inclusion that decreased significantly to $3.3 \pm 0.2$ after 6 months. A majority of the patients (55\%) had any type of changes on HRCT either focal or diffuse. Lung changes of any type were more frequent in RA as compared to non-RA individuals $(35 \%)$ with a higher prevalence of lung fibrosis (15/82 in the RA cohort and $0 / 43$ in the control cohort). In the RA cohort one patient $(1.2 \%)$ was diagnosed with lung cancer following HRCT screening while none in the control cohort. ACPA and rheumatoid factor but not smoking status associated with the presence of changes on HRCT. Immunohistochemistry demonstrated a significant increase in expression of both PAD2 and PAD4 in the lungs of current smokers independent of the ACPA status with no significant changes following 6 months of treatment.

Conclusion Presence of ACPA associates with early lung HRCT changes in RA. The authors suggest that smoking (and other yet unidentified factors) promotes site specific citrullination (such as in lungs) leading to generation of ACPA immunity and lung changes very early in the disease process.

\title{
11 LUNG CHANGES DETECTED BY HIGH RESOLUTION TOMOGRAPHY ARE PRESENT IN ACPA POSITIVE RA PATIENTS ALREADY AT DISEASE ONSET
}

G Reynisdottir, V Joshua, S Nyren, A Hensvold, R Karimi, A Harju, M Engström, M Sköld, A Eklund, J Grunewald, L Klareskog, A I Catrina Rheumatology Unit, Department of Medicine, Karolinska University Hospital, Karolinska Institute, Stockholm, Sweden

\subsection{6/annrheumdis-2011-201231.11}

Background/purpose The authors have previously shown that smoking increases citrullination in the lungs of healthy smokers and hypothesised that this process is an important step in the early development of rheumatoid arthritis (RA). To test this the authors investigated lung function in a cohort of RA patients with very early disease, already at the time of diagnosis and compared it with an aged, smoke and gender matched cohort of individuals without RA.

Methods 82 patients, 56 female and 26 male, median age 60 (range 22-84) with symptom duration less than 1 year at the time of diagnosis and naive to disease-modifying antirheumatic drug treatment were included in the RA cohort. A second cohort of age, smoking and gender matched non-RA individuals $(n=43)$ were investigated with an identical protocol. Lung function (x-ray, high-resolution CT (HRCT) and dynamic spirometry) was tested in both RA and controls at baseline and repeated after 6 months in the RA group. In a subgroup of patients bronchoscopy was performed at inclusion $(n=18)$ and 6 months later $(n=11)$ and bronchoalveolar lavage samples as well as mucosal large bronchial biopsies were retrieved. Presence of peptydilamino deiminase (PAD) enzymes were evaluated by immunohistochemistry.

Results $63 \%$ of the patients were anticitrullinated protein antibodies (ACPA) positive, $26 \%$ were current smokers and $22 \%$ have reported a previous lung pathology. All patients had active disease with a mean disease activity score of $5.5 \pm 0.1$ at 